\title{
Pour une approche génétique de l'ébauche
}

For a genetic approach to the "ébauche"

\section{Floriane Philippe}

\section{OpenEdition Journals}

Édition électronique

URL : https://journals.openedition.org/cel/15115

DOI : 10.4000/cel.15115

ISSN : 2262-208X

\section{Éditeur}

École du Louvre

\section{Référence électronique}

Floriane Philippe, «Pour une approche génétique de l'ébauche », Les Cahiers de l'École du Louvre [En ligne], 16 | 2021, mis en ligne le 24 mai 2021, consulté le 30 juillet 2021. URL : http:// journals.openedition.org/cel/15115 ; DOI : https://doi.org/10.4000/cel.15115

Ce document a été généré automatiquement le 30 juillet 2021.

\section{(c) $($ i) $(9)$}

Les Cahiers de l'École du Louvre sont mis à disposition selon les termes de la licence Creative Commons Attribution - Pas d'Utilisation Commerciale - Pas de Modification 4.0 International. 


\title{
Pour une approche génétique de l'ébauche
}

\author{
For a genetic approach to the "ébauche"
}

Floriane Philippe

1 La langue française possède un lexique artistique à la fois riche et précis, notamment pour qualifier les étapes et les documents de la création: carton, crayon, croquis, dessin préparatoire, esquisse (première ou définitive), étude (de détail ou d'ensemble), modèle, etc. Cependant, dans l'usage, les significations de ces termes ne sont pas toujours clairement établies ou connues et suscitent des confusions, y compris chez les chercheurs, les professionnels du patrimoine et les collectionneurs. Ces difficultés de terminologie se rencontrent par exemple lors de l'identification d'une œuvre d'art pour un catalogue ou une base de données: tel dessin doit-il être désigné comme " croquis ", comme " étude " ou comme " esquisse "? Le cas du terme " ébauche " est particulièrement significatif. Il s'agit d'un mot d'usage courant, issu du vocabulaire artistique, qui n'a pourtant jamais fait l'objet d'une étude spécifique en histoire de l'art ${ }^{1}$, et dont la signification même est incertaine ou inconnue. Malgré des définitions d'autorité qui établissent de manière claire, dès le XVIII ${ }^{e}$ siècle, la distinction entre les deux termes, "ébauche » est souvent encore considéré à tort comme un synonyme d'« esquisse ».

2 Dans le catalogue Exquises esquisses (2017), publié dans le cadre d'une exposition au musée Magnin de Dijon, l'ancien conservateur de musée Patrick Ramade publie un article intitulé "L'esquisse peinte: extension du domaine d'étude », dans lequel il évoque indifféremment des esquisses, des ébauches et des œuvres inachevées ${ }^{2}$. Le commissaire de l'exposition Rémi Cariel précise dans le même ouvrage, dans un article consacré à l'esquisse dans la critique d'art :

«Si nous utilisons tantôt le terme d'esquisse tantôt celui d'ébauche, c'est que du point de vue de leurs qualités formelles et du jugement porté sur elles, en tant qu'on les distingue des œuvres achevées, il n'y a pas de différence essentielle ${ }^{3}$. »

3 La confusion relève donc à la fois d'une méconnaissance contemporaine et d'une habitude ancienne, car Rémi Cariel écrit alors sur le XIX siècle. Cet amalgame touche 
aussi les bases de données d'œuvres comme la base Joconde. Pour le mot-clef " ébauche ", le moteur de recherche propose par exemple un tableau intitulé Le Déluge (ébauche), alors que la description de la notice indique « esquisse pour le concours de Rome ». À l'inverse, une Esquisse pour l'Oratorio de Maurice Denis est qualifiée en description d'" ébauche au fusain, détrempe et gouache ".

La confusion entourant l'usage du terme " ébauche " est révélatrice des difficultés rencontrées par les historiens et historiennes de l'art pour qualifier les objets situés en marge de la création artistique proprement dite, dans l'antériorité ou la périphérie de l'œuvre, c'est-à-dire dans sa genèse. Depuis quelques années, l'étude des produits et des traces du processus créatif a été entreprise par une discipline nouvelle, la génétique artistique 4 . La " critique génétique ", née au CNRS dans les années 1970, repose sur une méthode d'analyse des manuscrits des écrivains pour étudier le processus de création des œuvres. Elle possède son propre laboratoire depuis 1982, l'Institut des textes et manuscrits modernes (ITEM) ${ }^{5}$. Les possibilités d'application des outils de la génétique au domaine des arts plastiques et aux corpus non textuels sont explorées dès le début des années 1990. Ces recherches ont été publiées dans plusieurs numéros spéciaux de la revue Genesis : " Architecture » en 2000, "Formes » en 2004, " Photographies » en 2015, « Bande dessinée » en 2016. Les objets d'études de la génétique littéraire sont les manuscrits, les brouillons, les notes des écrivains; ceux de la génétique artistique, qui documentent la création plastique, sont les les dessins préparatoires, les esquisses, les études, les carnets, journaux et notes autographes des artistes. L'approche génétique est précieuse pour aborder un objet d'étude original comme l'ébauche. La reconstitution du processus de création d'une œuvre suppose une exigence d'exhaustivité qui conduit à prendre en compte des documents habituellement marginalisés par l'histoire de l'art pour leur peu de valeur esthétique (un modeste croquis, de légères annotations) ou pour leur caractère inabouti, comme à porter un regard nouveau sur des productions amplement connues et mises en valeur (esquisses peintes, études de nu ou de visage). L'un des enjeux premiers et nécessaires de la génétique des arts plastiques est d'élaborer un lexique précis qui permette la description des objets et des processus qu'elle étudie.

\section{Histoire d'une ambiguiité}

5 Le vocabulaire artistique français s'est constitué au cours du XVII ${ }^{e}$ siècle, mais ce n'est qu'au siècle suivant qu'il est véritablement fixé. En principe, la différence entre ébauche et esquisse est clairement établie dès le XVIII siècle au moins. L'ébauche constitue le commencement de l'exécution de l'œuvre elle-même, tandis que l'esquisse est un travail préparatoire (dessiné, peint ou sculpté). L'ébauche appartient à la matière même de l'œuvre alors que l'esquisse est réalisée sur un support indépendant. L'Encyclopédie (1755) introduit cette distinction dans l'article " Ébauche, esquisse », qui met les deux pratiques en opposition pour mieux souligner leurs spécificités : l'ébauche est définie comme la " première forme " d'une œuvre, l'esquisse comme le modèle de cette œuvre ${ }^{6}$. L'article de Levesque pour l'Encyclopédie méthodique (1788) reprend cette définition en affirmant dès la première ligne au sujet de l'ébauche que " ce terme n'est pas synonyme d'esquisse ${ }^{7} »$. Pourtant, un siècle plus tard, Larousse (1870) doit mettre en garde contre les erreurs de certains dictionnaires, y compris parmi les plus réputés : 
" L'Académie elle-même a confondu ces deux objets, faciles cependant à distinguer." "

«L'ébauche n'est pas, comme le prétendent certains dictionnaires et certaines encyclopédies, la première exécution de l'idée de l'artiste, faite d'un jet, dans ce qu'on appelle le moment de l'inspiration, c'est-à-dire lorsqu'il est bien possédé par son sujet et qu'il a la vision de son ensemble. C'est là l'esquisse, mais non l'ébauche ${ }^{9}$."

6 Le Dictionnaire de l'Académie française ( $4^{\mathrm{e}}$ édition) avait en effet donné en 1762 une définition de l'ébauche succincte et conforme à celle de l'Encyclopédie, mais l'article « esquisse » présentait dans sa définition le terme " ébauche » comme un synonyme : "Ébauche, premier crayon de quelque ouvrage que le Peintre médite de faire ${ }^{10}$. " La formulation de l'Académie, presque contemporaine de l'Encyclopédie, était en fait très proche de celle adoptée par Félibien un siècle plus tôt (1676). Celui-ci décrivait l'esquisse comme : « [...] une légère ébausche ou le premier crayon de quelque pensée \& de quelque ouvrage qu'on médite de faire ${ }^{11}$. " Ces deux textes rappellent qu'en français, le mot « esquisse » a d'abord désigné des dessins, avant que sa signification soit étendue aux productions peintes et sculptées.

7 «Ébauche » est le déverbal de " ébaucher ", issu de l'ancien picard " esbosquier » (XIV ${ }^{\mathrm{e}}$ siècle) qui signifie " ébrancher un arbre » (pour en faire une poutre, bauch, en ancien français). D’où le sens de "dégrossir " un objet, commencer sa fabrication. Il est d'abord utilisé dans le domaine de la sculpture, puis de la peinture (fin du $\mathrm{XV}^{\mathrm{e}}$ siècle $)^{12}$. Le Trésor de la Langue française atteste à partir de 1619 le mot esbauche au sens de " première forme d'une œuvre d'art " ${ }^{13}$. Le terme " esquisse " apparait plus tardivement en français. Il est issu de l'italien schizzo, du verbe schizzare, « jaillir, gicler " et désigne initialement " la tache que fait un liquide qui gicle ${ }^{14}$. Comme l'écrit Félibien en 1676, ce terme qualifie à l'origine des dessins :

«Et parce que les Ouvriers font ces premiers desseins avec furie \& promptitude d'esprit, \& en peu de temps, les Italiens ont nommé cela Squizzi, de Squizzare, qui veut dire sortir dehors, \& jalir [sic] avec impétuosité ${ }^{15}$. »

8 Le terme s'est diffusé au XVII ${ }^{e}$ siècle en français et dans d'autres langues européennes pour désigner les peintures préparatoires à une œuvre, pour lesquelles il n'existait auparavant pas de dénomination ${ }^{16}$.

9 La même complexité et la même confusion se rencontrent dans d'autres langues européennes quand on cherche à traduire le terme " ébauche ». En allemand, le terme " Entwurf ", du verbe " entwerfen ", est proche du français " ébauche ", mais comme dans notre langue, sa distinction avec les mots "Skizze " et son verbe "skizzieren ", équivalent de notre « esquisse ", n'est pas claire. En italien, " ébauche " peut être traduit par « abbozzo ", dont l'étymologie est similaire (dérivé d'abbozzare, issu de bozza, la bosse). Le terme connaît la même évolution que son équivalent français. Avec la naissance de la pratique de l'esquisse peinte, les termes " abbozo ", " sbozzo » et " bozza " en sont venus à désigner non plus seulement des œuvres au commencement de leur exécution ou laissées inachevées, mais aussi des travaux préparatoires à l'huile d'œuvres à venir, ou des répliques, ce qu'on désigne communément en français sous le terme d' " esquisse ${ }^{17}$. Aujourd'hui, c'est le terme "bozzetto " qui est communément employé pour qualifier les esquisses peintes. "Schizzo » est quant à lui circonscrit au domaine du dessin, tandis que " bozza » n'est plus utilisé que dans le contexte de l'édition (premier état d'un ouvrage). En anglais, l'emprunt de schizzo a donné naissance au terme générique de " sketch », qui 
désigne aussi bien les ébauches que les esquisses, les croquis, les études, etc. Il n'existe pas d'équivalent d'" ébauche " dans cette langue mais on peut donner une traduction satisfaisante d'« ébaucher " avec le verbe " to rough out ", dérivé de l'adjectif rough (grossier, irrégulier, brut, approximatif). Pour la peinture il existe cependant le terme " underpainting ", qui désigne les premières couches d'un tableau et ressemble au français « dessous ».

\section{Esquisse ou ébauche? Les origines de la confusion}

Contrairement à " esquisse ", le mot "ébauche » a acquis au cours du temps de nouvelles acceptions qui ont considérablement élargi sa signification hors du champ artistique. Dans le langage courant, il sert à dénoter l'imperfection d'une chose, matérielle ou non, ou à qualifier l'amorce d'un phénomène, de quelque nature qu'il soit. La définition du Trésor de la langue française rend compte de cette évolution. On constate que l'usage s'est étendu des productions matérielles aux créations de l'esprit, œuvres littéraires et travaux intellectuels. Mais surtout, de la signification originelle de " première forme, première façon donnée à une chose ", dérive un deuxième sens, par analogie: " premier indice, premier développement (d'une chose en devenir) ", qui concerne plus particulièrement les opérations de l'esprit. On donne pour exemple " l'ébauche d'un projet ». L'extension de l'usage du terme aux processus intellectuels autorise un glissement de sens qui étend considérablement sa signification : l'ébauche peut désigner aussi bien les premières étapes de l'exécution d'une œuvre que les premiers moments de la formulation d'une idée, d'un programme ou d'un texte. Ce qui en fait un quasi synonyme d'" esquisse ». Ces usages sont anciens. Furetière (1690) mentionne le sens littéraire d'" ébauche » et définit " ébaucher » comme: «Tracer grossièrement quelque ouvrage, en attendant qu'on le finisse; jeter ses premières pensées sur le papier ${ }^{18}$. "

" ESBAUCHE. s. f. Les premiers traits d'un tableau; les premières pensées sur un ouvrage ; le premier dessein d'un bastiment, \& autres choses qui se finissent ou se polissent avec le temps, après avoir été d'abord grossièrement tracées. Une légère esbauche d'un grand Peintre vaut souvent mieux que les ouvrages finis d'un autre. On doit faire une esbauche d'un Poëme Dramatique, en distribuer le sujet, avant que d'en faire les vers ${ }^{19}$."

11 Les différences entre " ébauche » et " esquisse » sur le plan de l'étymologie peuvent aussi en partie expliquer la confusion. L'esquisse est définie par l'Académie française et par Félibien en référence à l'ébauche, probablement parce qu'il s'agit d'un mot nouveau, emprunté à une langue étrangère. La comparaison est peut-être aussi encouragée par le fait que ces termes, s'ils appartiennent tous deux au champ du vocabulaire artistique, concernent originairement des media différents. Chez Félibien comme dans la première édition du Dictionnaire de l'Académie, l'esquisse est définie comme " premier crayon ».

Dans les textes du XIXe siècle, la distinction entre esquisse et ébauche est bien davantage respectée par les artistes que par les critiques d'art. Delacroix, dans les comptes rendus sur son travail et dans ses réflexions d'ordre technique, utilise le mot " ébauche " exclusivement pour qualifier les premières couches d'un tableau en cours d'exécution :

" Je me suis mis, après mon déjeuner, à reprendre le Christ au tombeau : c'est la troisième séance d'ébauche; et, dans ma journée, malgré un peu de malaise, je l'ai 
remonté vigoureusement et mis en état d'attendre une quatrième reprise. Je suis satisfait de cette ébauche, mais comment conserver, en ajoutant des détails, cette impression d'ensemble qui résulte de masses très simples ${ }^{20}$ ? "

Les manuels d'apprentissage de la peinture sont aussi fidèles à la définition que donnait Diderot. Goupil par exemple enseigne que :

"L'ébauche se fait par-dessus le dessin, elle indique les effets à obtenir et distribue les couleurs que doivent revêtir les différentes parties de la composition, ou du moins elle en prépare les dessous. Enfin, c'est par-dessus l'ébauche qu'on termine $[\ldots]^{21} . »$

Il faudrait signaler la distinction entre un usage technique, pratique, de ce vocabulaire par les artistes, et un usage métaphorique, par la théorie et la critique d'art. Le mot " ébauche " est employé pour dénoter un style ou les qualités esthétiques d'une œuvre, par exemple chez Charles Blanc :

« C'est surtout dans ses ébauches qu'on sent le peintre habile, par exemple dans les deux esquisses, d'un beau jet et d'une belle couleur, qui font partie de la précieuse galerie de lord Overstone ${ }^{22}$. "

Ou chez Théophile Thoré :

" C'est une large ébauche, magistralement enlevée sur des frottis roux, [...] tout est à l'effet dans cette esquisse qui pourrait bien avoir été prise d'après nature, ou qui certainement a été jetée de premier coup sur la toile, dans un moment d'inspiration ${ }^{23}$."

L'ébauche est apparentée à l'esquisse pour ses caractéristiques plastiques: une apparence de spontanéité, une touche large et " lâchée ", une expressivité renforcée par l'imprécision des formes.

Terme technique ou concept esthétique, le statut de l'ébauche change en fonction du discours. Le mot prend une valeur toute particulière dans le contexte des débats autour de la notion de fini. À partir du printemps de l'année 1853, Delacroix développe dans son Journal des réflexions théoriques sur l'inachèvement, s'intéressant à " l'effet que produisent les choses inachevées: esquisses, ébauches, etc ${ }^{24}$. " La distinction entre esquisse et ébauche se dissout, les deux étant traitées comme les objets d'une même classe, définie par opposition à l'œuvre achevée. Delacroix peut ainsi écrire que " l'artiste ne gâche donc pas son tableau en le finissant; seulement, en renonçant au vague de l'esquisse, il se montre davantage dans sa personnalitée ${ }^{25}$. "

Le qualificatif d'« ébauche » sera appliqué par la critique d'art tantôt en un sens positif pour souligner les qualités expressives d'une œuvre, comme dans les extraits de Blanc et de Thoré, tantôt de manière péjorative pour dénoter une œuvre insuffisamment finie, par exemple chez Théophile Gautier à propos d'un tableau de Chassériau :

"Ses Cavaliers arabes, s'injuriant avant le combat à la façon homérique, auraient prêté à un développement de nobles formes, d'attitudes superbes, de draperies au grand jet que l'artiste a négligé pour une turbulence d'esquisse, une furie de brosse qui montrent la préoccupation d'une autre manière; des incorrections voulues, une touche heurtée, des oppositions violentes de ton, donnent à cette toile l'aspect d'une ébauche plutôt que d'un tableau $[. . .]^{26}$. "

Le terme vient signaler l'état d'inachèvement de l'œuvre et par conséquent lui dénie son statut même d'œuvre d'art: une ébauche plutôt qu'un tableau. L'ébauche est souvent caractérisée par des expressions négatives, définie par ce qu'elle n'est pas: c'est un ouvrage grossier, non fini, sommaire, etc. Autant de qualifications qui peuvent s'appliquer à d'autres types d'objets, en arts plastiques (esquisse, étude, croquis) ou en 
littérature et autres activités intellectuelles (brouillons, plans, projets). En 1762 l'Académie la définissait comme :

« Ouvrage de Peinture \& de Sculpture, qui n'est que grossièrement commencé. Ce n'est qu'une légère ébauche, que la première ébauche. Il se dit figurément des ouvrages d'esprit. Cette pièce n'est pas achevée, elle n'a pas la dernière main, ce n'est qu'une ébauche ${ }^{27}$. "

"Ébauche » comme " esquisse " n'ont jamais été seulement des termes techniques qualifiant une pratique ou un produit du processus de création. Étymologiquement, l'ébauche désigne une activité particulière, celle du dégrossissage d'un objet. Le terme " esquisse » en revanche ne qualifie pas un type d'action mais une qualité, une manière d'agir. Le schizzo est le produit d'un geste vif, spontané, capable de traduire les fulgurances de l'inspiration ${ }^{28}$. Son identité repose en grande partie sur des critères esthétiques, autorisant des rapprochements avec d'autres œuvres au statut génétique différent mais d'aspect similaire: Selon Christian Michel, « elle réunit les signes de la première et de la troisième étape d'élaboration du tableau : l'ébauche, dans laquelle le peintre montre son jeu, et les retouches, où il montre son esprit ${ }^{29}$. " Tandis que pour Patrick Ramade, "les distinctions émises fonctionnent grosso modo jusqu'au $\mathrm{XVIII}^{\mathrm{e}}$ siècle, époque à partir de laquelle la conception de l'esquisse passe d'une fonction à une manière $e^{30}$."

\section{L'ébauche, un objet spécifique pour l'histoire de l'art?}

21 On peut conclure de ces péripéties historiques et des complexités de la notion que l'étude de l'ébauche en art implique de dépasser les seuls enjeux esthétiques et théoriques au profit d'une approche génétique. Les usages métaphoriques du terme " ébauche » ont tout à fait leur place dans une histoire de la théorie de l'art, mais ils peuvent aussi prêter à confusion, et pour les besoins d'une analyse des processus de création, il paraît nécessaire de disposer d'un vocabulaire clair et précis.

L'ébauche est un objet d'étude qui ne va pas de soi et qui peut sembler paradoxal. Elle constitue dans la plupart des cas la part inaccessible du processus de création, celle qui ne laisse pas de traces. Contrairement aux documents préparatoires, aux dessins, aux notes, aux esquisses, l'ébauche est par nature destinée à disparaître (en sculpture : arte di levare), à se laisser recouvrir (en peinture : arte di porre) pour permettre l'émergence de l'œuvre achevée. Quand elle est conservée, c'est souvent par accident, ou en raison d'un échec ou d'une suspension du processus créatif : mort de l'artiste, abandon du projet, échec de l'exécution. Même dans le cadre des études génétiques, l'ébauche occupe une place à part. Elle ne fait pas partie du dossier de genèse au même titre que les travaux préparatoires, car elle ne peut en principe pas être comparée avec l'œuvre achevée. Comme le précise Gérard Genette : «Une ébauche abandonnée peut fort bien constituer un objet artistique (en tout cas, esthétique) en elle-même, mais elle ne témoigne de rien d'autre $[. . .]^{31}$. » Elle appelle donc une méthodologie spécifique, ne serait-ce que pour rendre interprétable l'interruption ou le renoncement dont elle est la trace. 
Figure 1.

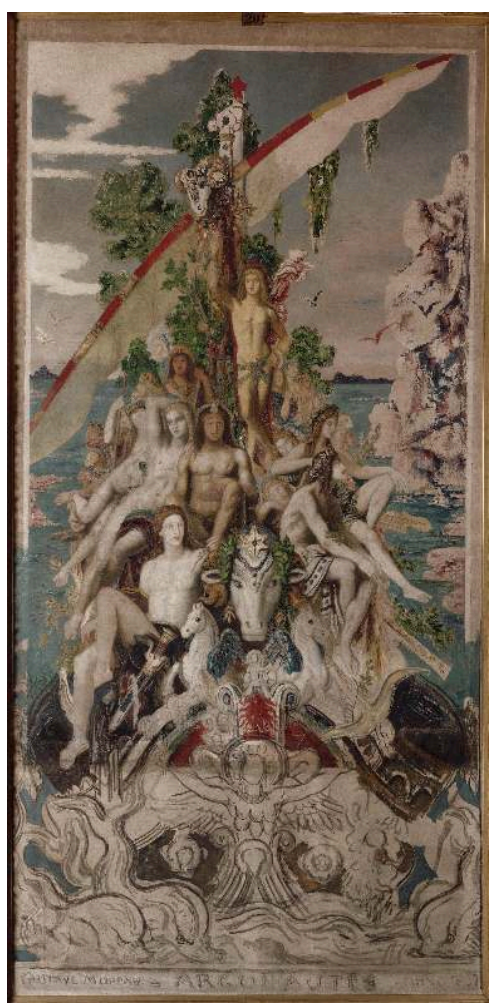

Gustave Moreau, Le Retour des Argonautes, huile sur toile, 4,34 x 2,15 m., Paris, Musée Gustave Moreau, Cat. 20, vers 1891-1897.

(c) RMN-Grand Palais / René-Gabriel Ojéda

Le souvenir d'une ébauche peut être conservé dans les notes d'un artiste, comme celles de Delacroix dans le Journal, ou par le témoignage d'un observateur. Dans L'Atelier d'Ingres: souvenirs d'Amaury-Duval, un chapitre relate la visite de l'élève dans l'atelier du maitre, avec des descriptions de plusieurs œuvres en cours d'exécution, dont la Vénus anadyomène (1808-848) et La Source (1820-1856) dans leur premier état.

« Le ciel était d'un ton bleuâtre plutôt que bleu; toute la figure avait cet aspect si attrayant de l'ébauche, les Amours à peine indiqués mais charmants. [...] Depuis, qu'est-il arrivé ? Aujourd'hui, le ciel est d'un bleu foncé, presque noir, sur lequel la Vénus se détache en lumière vive, et, quand je l'ai vue pour la première fois, le passage du ton du ciel à celui de la figure était à peine sensible. M. Ingres avait-il perdu cette naïveté qu'il me vantait, lorsqu'il acheva ce tableau commencé dans sa jeunesse ${ }^{32}$ ?"

Pour l'époque contemporaine, on dispose parfois d'enregistrements photo ou vidéo, dont l'archétype est le film Le Mystère Picasso de Henri-Georges Clouzot (1956), même si le principe de cette mise en scène de la genèse de l'œuvre reste relativement factice. Le catalogue de l'exposition Gustave Moreau de 1998 contient plusieurs "Dossiers " décomposant les étapes de la création de certaines œuvres à partir de différents types de documents. Le dossier Jupiter et Sémélé s'appuie notamment sur des photographies du tableau conservées au Musée Gustave Moreau, mais aussi sur des analyses menées par le Laboratoire des Musées de France. Les techniques d'imagerie scientifique comme la radiographie et la réflectographie infrarouge, couplées aux analyses chimiques des matériaux, permettent d'étudier les couches inférieures des œuvres en deux dimensions, notamment pour déceler des changements de composition. Ces méthodes 
sont cependant insuffisantes en elles-mêmes pour reconstituer l'aspect de l'ébauche et doivent être complétées par des études historiques et l'observation à l'œil nu. Parfois l'ébauche reste visible au sein même de l'œuvre, soit dans des zones laissées inachevées, pour des raisons esthétiques (non finito) ou techniques (traitement des ombres en transparence, effets de perspective, etc.), soit par transparence, pour les œuvres dont l'exécution procède par superposition. Dans le cas de la peinture à l'huile, l'ébauche est souvent partie intégrante du coloris du tableau et entre dans la composition des tons les plus subtils ${ }^{33}$.

C'est dans les œuvres inachevées que l'ébauche est le plus immédiatement accessible.

Elles permettent par comparaison d'étudier les techniques de création d'un artiste, voire d'un groupe d'artistes, les manières d'ébaucher variant en fonction des mouvements artistiques ou des choix individuels. Chez un peintre comme Gustave Moreau, qui a laissé un grand nombre d'œuvres inachevées, elles offrent des informations très complètes sur les processus de création de l'artiste. Dans une lettre à Eugène Fromentin en 1856, à propos du tableau Le Jeune Homme et la Mort (1865), il décrit sa manière d'ébaucher :

«[...] je commence par le plus difficile, la ligne et le modelé intérieur, que j'accentue très vigoureusement et que je couvre ensuite avec grande liberté de fluidités colorées, balayant le tout sans crainte et traitant la chose en esquisse ${ }^{34}$. »

Figure 2.

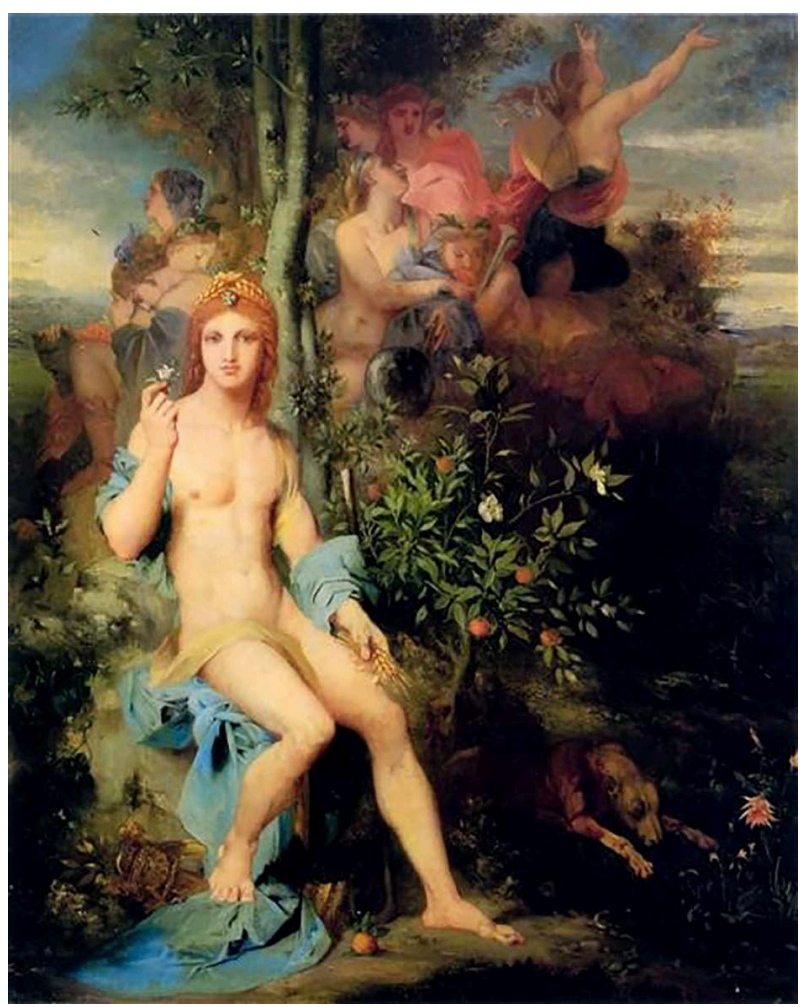

Gustave Moreau, Apollon et les Neuf Muses, huile sur toile, 0,83 x 1,03 m., collection particulière, 1856. 
Figure 3.

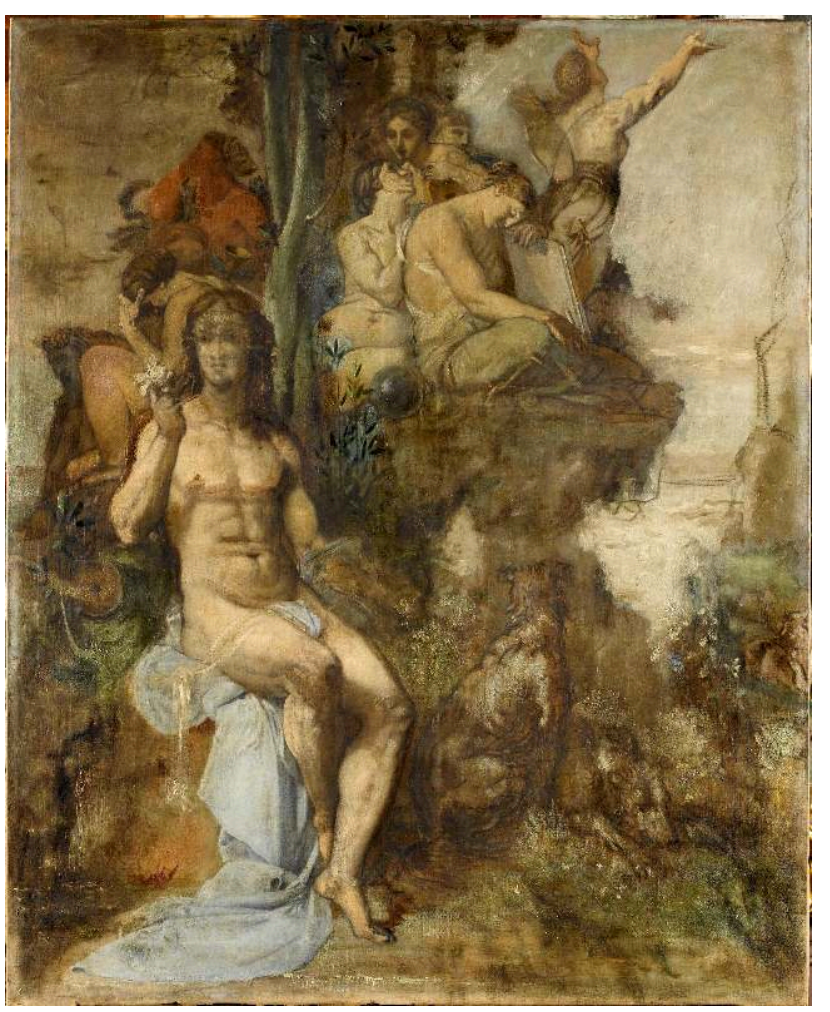

Gustave Moreau, Apollon et les Muses, huile sur toile, 100 × 80 cm, Paris, Musée Gustave Moreau, Cat. 609, vers 1856.

(c) RMN-Grand Palais / René-Gabriel Ojéda

On peut observer cette technique mise en œuvre dans un tableau inachevé de la même époque, Apollon et les Muses, où les figures et le paysage sont indiqués par un épais cerne noir et les zones d'ombre et de lumière par un lavis d'huile sépia et blanc ${ }^{35}$. L'œuvre entière est à l'état d'ébauche, à l'exception du corps d'Apollon et du drapé blanc. Si les corps sont dessinés avec précision, les éléments de décor végétaux et minéraux sont laissés dans une forme d'indétermination. Il existe une version achevée ${ }^{36}$ de ce même tableau, conservé dans une collection particulière, qui permet de juger des différences significatives entre les deux réalisations. D'autres tableaux comme Le Retour des Argonautes (v. 1891-1897) témoignent d'une technique différente où le dessin occupe une plus grande place et où chaque morceau est traité séparément ${ }^{37}$. Dans certaines œuvres, Moreau adopte une démarche expérimentale où la composition est créée par la couleur, souvent empâtée, et où l'exécution laisse place à une forme d'improvisation. Thomyris et Cyrus est un tableau pour lequel on ne connaît pas de dessin d'ensemble; il s'agit d'un grand paysage avec quelques figures, laissé inachevé ${ }^{38}$. Le peintre a procédé par superpositions ou par recouvrements, avec le pinceau, le couteau ou même le doigt, et l'on comprend que la toile a subi plusieurs transformations, sans qu'il soit possible de déterminer quel a été le point de départ. 
Figure 4.

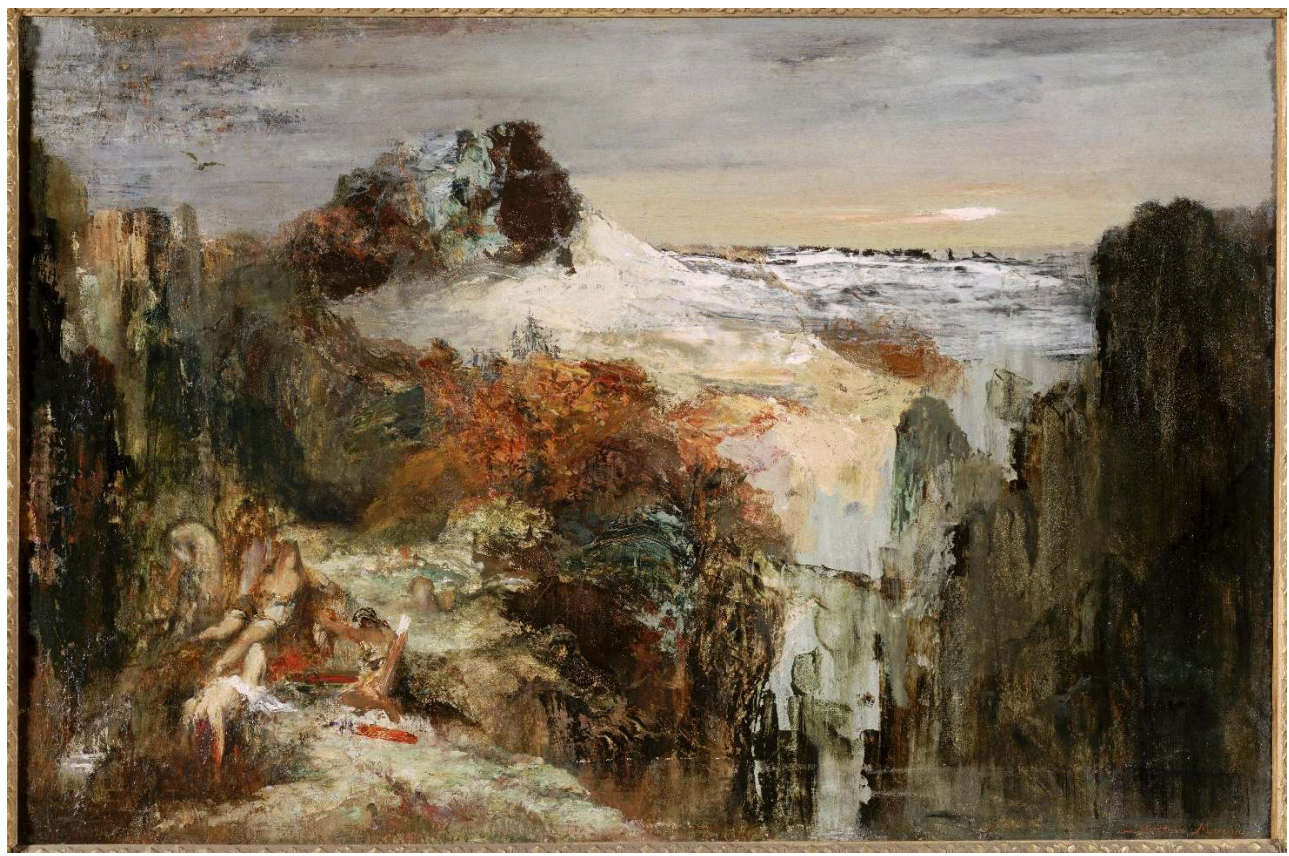

Gustave Moreau, Thomyris et Cyrus, huile sur toile, 0,57 × 0,87 m., Paris, Musée Gustave Moreau, Inv. 13978.

(c) Gérard Blot / Réunion des musées nationaux

27 Les œuvres inachevées, en particulier celles qui possèdent la complexité de Thomyris et Cyrus, interrogent la définition de la catégorie " ébauche ». Dans certains cas, il s'agit d'une étape technique définie du processus de création, avec un terme identifié. Dans l'enseignement académique de la peinture à l'huile, l'ébauche est séparée du reste de l'exécution par un temps de séchage:

"Un mois dans un atelier bien sec ou une quinzaine de jours par un plein soleil d'été suffisent parfaitement. Quand l'ébauche sera bien sèche, on devra passer une couche générale de vernis à retoucher sur tout le tableau avant de le reprendre ${ }^{39}$. "

En raison de cette technicité, l'étape de l'ébauche peut être confiée à des assistants, des élèves, ou des praticiens dans le cas de la sculpture de taille. La division du temps d'exécution correspond alors à la division du travail au sein de l'atelier. Chez d'autres artistes, la phase d'ébauche est beaucoup plus longue et cette limite moins évidente : au lieu d'être traitée d'un seul mouvement, elle peut faire l'objet d'un processus comportant plusieurs phases, et il devient alors plus difficile de préciser où s'arrête véritablement l'ébauche et à quel moment l'artiste entre à proprement parler dans l'exécution finale. C'est le cas chez Delacroix, comme le montrent ses notes de travail où il décrit l'élaboration d'ébauches composées de plusieurs couches de couleur :

" La Femme impertinente était préparée très empâtée et d'un ton très chaud et surtout très rouge. Passé dessus un glacis de terre verte, peut-être un peu de blanc. Cela a fait la demi-teinte gris opale irisée ; là-dessus touché simplement des clairs avec l'excellent ton terre Cassel, blanc et un peu de vermillon; puis quelques tons orangés francs par places. Tout ceci n'était encore qu'une préparation, mais de la plus grande finesse ${ }^{40}$. "

L'ébauche n'est pas une étape nécessaire à toute œuvre d'art, notamment en peinture. Des œuvres peuvent être entièrement ébauchées, ou seulement par parties; certains artistes ne font d'ailleurs pas du tout d'ébauche (on parle de " premier jet»). En 
peinture, il s'agit d'une pratique qui remonte sans doute à la Renaissance mais qui a connu des formes très variables, avant de disparaître assez largement avec l'impressionnisme et la peinture contemporaine.

En conclusion, l'ébauche se présente donc comme un élément constitutif de la phase d'exécution mais aussi l'étape intermédiaire entre la conception de l'œuvre (études préparatoires, esquisses) et son achèvement, ce qui lui confère un rôle décisif, et même crucial, dans le processus de création. L'ébauche témoigne ainsi d'une dynamique d'achèvement ou au contraire permet le constat d'un échec, d'un blocage ou d'un ajournement. Dans tous les cas il s'agit d'un objet aux contours incertains, doté d'une double dimension matérielle et métaphorique, qui possède un statut particulier au carrefour d'enjeux théoriques, historiques, esthétiques et génétiques. Le travail de définition amorcé dans cet article doit être poursuivi pour faire de l'ébauche une catégorie vraiment opérante en histoire de l'art. Il existe différentes méthodes d'enregistrement des étapes de l'exécution, nous l'avons rappelé, mais l'interprétation correcte d'une œuvre inachevée, d'une photographie ou d'une radiographie suppose une compréhension fine à la fois de l'étendue et des limites de la notion d'ébauche. Cet article entend poser les bases d'un champ d'études encore largement inexploré qui intéresse aussi bien la génétique que l'étude matérielle des œuvres et l'histoire de l'art en général. L'ébauche met au jour les choix esthétiques de l'artiste ainsi que son rapport à l'inachèvement, ses méthodes d'exécution et la part de technicité ou d'improvisation à l'œuvre dans celles-ci ; elle permet de saisir toutes les évolutions de la composition d'une œuvre. Cette étude, préliminaire à une recherche doctorale consacrée à l'esquisse et l'ébauche dans la création picturale romantique, prend place dans un travail collectif plus étendu, mené au sein de l'ITEM, sur le vocabulaire artistique. Ce projet nommé DIGA (Données Internationales de Génétique Artistique) a pour objectif la création d'un dictionnaire terminologique sous forme de base de données accessible en ligne.

\section{NOTES}

1. Contrairement à l'esquisse. Plusieurs expositions ont été consacrées aux esquisses en France et aux États-Unis, depuis 1967 et Masters of the loaded brush, dont le catalogue d'exposition est introduit par Rudolph Wittkower. Pour la France on peut citer L'apothéose du geste (Strasbourg et Tours, 2003), Esquisses peintes de l'époque romantique (Paris, 2013) et Exquises esquisses (Dijon, 2018). D'autres catalogues explorent la question des œuvres inachevées et de l'inachèvement en peinture. Je nommerai seulement celui de Nico Van Hout, The Unfinished painting (2012).

2. Patrick Ramade, «L'esquisse peinte: extension du domaine d'étude », Rémi Cariel (dir.), Exquises esquisses: du projet à la réalisation, Paris, Réunion des Musées nationaux, 2017, pp. 11-24.

3. Rémi Cariel, "Où s'arrête l'esquisse, où commence l'œuvre? L'esquisse et la manière d'esquisse dans la critique d'art au XIX ${ }^{\mathrm{e}}$ siècle », ibid., pp. 47-72, p. 47.

4. Sur la naissance et le développement de la discipline, voir Pierre-Marc de Biasi, «Génétique des arts plastiques ", Littérature, $\mathrm{n}^{\circ}$ 178, 2015/2, pp. 64-79; P.-M. de Biasi, «Pour une génétique généralisée: l'approche des processus dans les arts et les sciences", P.-M. de Biasi et Anne 
Herschberg Pierrot (dir.), L'Eeuvre comme processus, Paris, CNRS Éditions, 2017, pp. 289-305. Un numéro spécial de la revue Genesis a été consacré aux arts plastiques: "Formes ", Genesis (Manuscrits-Recherche-Invention), $\mathrm{n}^{\circ} 24,2004$.

5. Le texte fondateur de la critique génétique paraît en 1979: Aragon, Jean Bellemin-Noël, Bernard Brun et al., Essais de critique génétique, Paris, Flammarion, 1979.

6. Denis Diderot, «Ébauche, esquisse", Denis Diderot et Jean Le Rond d'Alembert (dir.), Encyclopédie, ou Dictionnaire raisonné des Sciences, des Arts et des Métiers, Paris, Briasson, David, Le Breton et S. Faulche, vol. 5, 1755, pp. 212-213.

7. Pierre-Charles Levesque, «Ébauche », Pierre-Charles Levesque et Claude-Henri Watelet (dir), Encyclopédie méthodique. Beaux-arts, Paris, Panckoucke, vol. 1, 1788, pp. 206-207.

8. Pierre Larousse, «Esquisse », Grand dictionnaire universel du XIX ${ }^{e}$ siècle, Paris, Administration du grand Dictionnaire universel, t. 7, 1870, p. 934.

9. P. Larousse, «Ébauche », ibid., p. 36.

10. Académie française, "Esquisse", Dictionnaire de l'Académie française, Paris, Vve B. Brunet, 1762 , t. 1, p. 667.

11. André Félibien, Des principes de l'architecture, de la sculpture, de la peinture, et des autres arts qui en dépendent: avec un Dictionnaire des termes propres à chacun de ces arts, Paris, Jean-Baptiste Coignard, 1676, p. 581.

12. "Ledit compaignon sera tenu achecter et avoir agreable ce que les maistres lui ordonneront par escript pour faire sondit chef-d'euvre, et fera faire son tableau de bon boys bien sec, et sera encolé et blanchy bien et deuement, et puis pourtraict et ébauché de coulleurs à huyle et achevé de bonnes et loyalles coulleurs, et à la fin bien verny comme l'euvre le requerra (Ordonn. rois Fr. P., t. 20, 1496, 564). » «Esbauscher », Dictionnaire du Moyen français [en ligne], Atilf [consulté le 27 avril 2019], http://www.atilf.fr/dmf/.

13. «Ébaucher », Trésor de la Langue française informatisé [en ligne], Atilf, [consulté le 27 avril 2019] http://stella.atilf.fr.

14. "Ébaucher ", Trésor de la Langue française informatisé [en ligne], ibid.

15. A. Félibien, Des principes de l'architecture, op. cit. note 11, p. 581.

16. Voir Linda Bauer et George Bauer, "Artists' inventories and the language of the oil sketch ", The Burlington Magazine, vol. 141, $\mathrm{n}^{\circ} 1158$ (sept. 1999), pp. 520-530.

17. Ibid., p. 521.

18. Antoine Furetière, "Esbaucher ", Dictionnaire universel contenant generalement tous les mots françois, tant vieux que modernes, \& les termes de toutes les sciences et des arts, La Haye, A. et R. Leers, 1690, n. p.

19. A. Furetière, «Esbauche ", Dictionnaire universel, ibid., n. p.

20. Eugène Delacroix, « $1^{\mathrm{er}}$ mars 1847 », Journal, 1893, rééd. Paris, Plon, 1996, p. 137.

21. Frédéric Auguste Antoine Goupil, Manuel complet et simplifié de la peinture à l'huile, suivi du traité de la restauration des tableaux, Paris, Desloges, 1858, p. 37.

22. Charles Blanc, "Augustus Wall Callcott», Histoire des peintres de toutes les écoles et de tous les temps, Paris, Renouard, 1861, vol. 1, p. 384.

23. Théophile Thoré, Musées de la Hollande. Musée Van der Hoop à Amsterdam et Musée de Rotterdam, Paris, Renouard, 1860, p. 141.

24. E. Delacroix, « 9 mai 1853 », Journal, op. cit. note 20, p. 340.

25. Id., « 20 avril 1853 », p. 330.

26. Théophile Gautier, «Salon de 1852 », La Presse, 25 mai 1852, p. 2. Les italiques sont un ajout de l'autrice.

27. Académie française, «Ébauche ", Dictionnaire de l'Académie française, op. cit. note 20, p. 574.

28. «En peinture, l'esquisse ne dépend en aucune façon des moyens qu'on peut employer pour la produire. [...] L'esquisse est la première idée d'un sujet de peinture. L'artiste qui veut le créer et dans l'imagination duquel ce sujet se monte sous différents aspects, risque de voir disparaître des 
formes qui se présentent en trop grand nombre, s'il ne les fixe par des traits qui puissent lui en rappeler le souvenir. Pour parvenir à suivre le rapide essor de son génie, il ne s'occupe point à surmonter les difficultés que la pratique de son art lui oppose sans cesse ; sa main agit, pour ainsi dire théoriquement; elle trace des lignes auxquelles l'habitude de dessiner donne à-peu-près les formes nécessaires pour y reconnaître les objets. L'imagination, maîtresse absolue de cet ouvrage, ne souffre qu'impatiemment le plus petit ralentissement dans la production. C'est cette rapidité d'exécution qui est le principe du feu qu'on voit briller dans les esquisses des peintres de génie. On y reconnaît l'empreinte du mouvement de leur âme; on en calcule la force et la fécondité. » C.-H. Watelet, « Esquisse », P.-C. Levesque et C.-H. Watelet, Encyclopédie méthodique, op. cit. note 7, pp. 267-268.

29. Christian Michel, «Imagination et feu, l'esquisse dans la pensée du XVIII ${ }^{\mathrm{e}}$ siècle », Dominique Jacquot (dir.), L'apothéose du geste. L'esquisse peinte au siècle de Boucher et Fragonard, Les Musées de Strasbourg, Musée des Beaux-Arts de Tours, Hazan, 2003, pp. 39-47, p. 43.

30. P. Ramade, art. cité note 2, p. 12.

31. Gérard Genette, L'Euvre de l'Art. Immanence et transcendance, Paris, Seuil, 1994, p. 218.

32. Amaury-Duval, L'Atelier d'Ingres : souvenirs, Paris, G. Charpentier, 1878, pp. 85-86.

33. «Une teinte préparée à l'ébauche d'un ton vineux peut devenir bleuâtre, en la recouvrant légèrement, à la seconde reprise, d'une couleur de chair locale où il n'entre point ou presque point de bleu: c'est ainsi que les habiles peintres ont fait apercevoir ces veines et ces tons bleuâtres qu'on voit si souvent à travers des peaux minces et délicates. » Pierre-Louis Bouvier, Manuel des jeunes artistes et amateurs en peinture, Paris, F.-G. Levrault, 1827, p. 327.

34. G. Moreau, lettre à Eugène Fromentin, 8 décembre 1856, Barbara Wright et Pierre Moisy (éd.), Gustave Moreau et Eugène Fromentin : documents inédits, La Rochelle, Quartier latin, 1972, p. 87.

35. G. Moreau, Apollon et les Muses, huile sur toile, 1,00 x 0,80 m., Paris, Musée Gustave Moreau, Cat. 609, vers 1856.

36. G. Moreau, Apollon et les Neuf Muses, huile sur toile, 0,83 x 1,03 m., collection particulière, 1856.

37. G. Moreau, Le Retour des Argonautes, huile sur toile, 4,34 x 2,15 m., Paris, Musée Gustave Moreau, Cat. 20, vers 1891-1897.

38. G. Moreau, Thomyris et Cyrus, huile sur toile, 0,57 x 0,87 m., Paris, Musée Gustave Moreau, Inv. 13978.

39. Johan Georges Vibert, La Science de la peinture, Paris, Paul Ollendorff, 1891, p. 191.

40. E. Delacroix, « Mardi 13 mai 1851 », Journal, p. 278.

\section{RÉSUMÉS}

Les termes « ébauche » et " esquisse » occupent une place centrale dans le lexique artistique. Cependant, leur usage est entouré d'une certaine confusion : leur signification reste peu claire et ils sont souvent traités comme des synonymes, y compris chez les historiens de l'art ou les professionnels des musées. Cet article propose une étude à la fois lexicale et matérielle de l'ébauche offrant les bases d'une définition opérante pour l'histoire de l'art. Il examine les causes de la confusion, en distinguant un usage technique du terme d'un emploi esthétique ou métaphorique. Comment et à quelles conditions l'ébauche peut-elle devenir objet d'étude pour l'histoire de l'art? Seule une approche génétique, centrée sur les processus de création, permet 
une compréhension fine et précise du phénomène de l'ébauche, sa fonction et sa place dans la genèse de l'œuvre.

The terms "ébauche" and "esquisse" occupy a central place in the French artistic lexicon. However, their use is surrounded by a certain amount of confusion : their meaning remains unclear, and they are often treated as synonyms, even by art historians and museum professionals. This article proposes a lexical and material study of the "ébauche" establishing the bases for a working definition for art historians. It examines the causes of the confusion, distinguishing a technical use of the term from an aesthetic or metaphorical one. How and under what conditions can the "ébauche" become an object of study for art history? Only a genetic approach, centred on the creative processes, allows a precise comprehension of the phenomenon of the "ébauche".

\section{INDEX}

Mots-clés : ébauche, esquisse, artistic genetic, creative processes, Gustave Moreau, artistic vocabulary, artistic lexicon, painting, genesis, epistemology

Keywords : Ébauche, esquisse, génétique artistique, processus de creation, Gustave Moreau, vocabulaire artistique, lexique artistique, peinture, genèse, épistémologie

\section{AUTEUR}

\section{FLORIANE PHILIPPE}

Doctorante à l'École du Louvre et Paris 1, Floriane Philippe étudie « L'esquisse et l'ébauche dans la création picturale romantique ». Elle travaille depuis 2016 comme stagiaire puis comme membre associée pour l'équipe « Histoire de l'art » de l'Institut des textes et manuscrits modernes (ENS/CNRS). Ses sujets de recherche concernent les processus de création artistique, la peinture et la théorie de l'art au XIXe siècle et l'histoire de la notion de patrimoine.

A doctoral student at the École du Louvre and Paris 1, Floriane Philippe is studying the "ébauche" and the "esquisse" in Romantic painting. She has been working for the "Art History" team of the Institut des Textes et Manuscrits Modernes (ENS/CNRS) since 2016, first an intern, then as an associate member. Her research topics concern the processes of artistic creation, painting and art theory in the nineteenth century, and the history of the concept of heritage. 